\title{
Facilitating dynamo action via control of large-scale turbulence
}

\author{
A. Limone, ${ }^{1, *}$ D.R. Hatch, ${ }^{1}$ C.B. Forest, ${ }^{2}$ and F. Jenko ${ }^{1}$ \\ ${ }^{1}$ Max Planck Institute for Plasma Physics, EURATOM Association, \\ Boltzmannstr. 2, 85748 Garching bei München, Germany \\ ${ }^{2}$ Department of Physics, University of Wisconsin, Madison, 1150 University Avenue, Madison, WI 53706, USA
}

\begin{abstract}
The magnetohydrodynamic dynamo effect is considered to be the major cause of magnetic field generation in geo- and astrophysical systems. Recent experimental and numerical results show that turbulence constitutes an obstacle to dynamos; yet its role in this context is not totally clear. Via numerical simulations, we identify large-scale turbulent vortices with a detrimental effect on the amplification of the magnetic field in a geometry of experimental interest, and propose a strategy for facilitating the dynamo instability by manipulating these detrimental "hidden" dynamics.
\end{abstract}

PACS numbers: 47.35.Tv, 07.55.Db, 47.65.-d, 95.30.Lz

Introduction.- The magnetohydrodynamic (MHD) dynamo effect is considered to be the main cause of the generation of self-sustained magnetic fields in geo- and astrophysical objects [1]: The kinetic energy of an electrically conducting fluid can be transformed into magnetic energy, $E_{M}$, as found analytically, experimentally, and numerically $[2,3,5,6]$. A magnetic field $\mathbf{B}$ can be generated in a conducting fluid (e.g., liquid metal or plasma) when the advection term in the governing induction equation sufficiently dominates the diffusion term in order to amplify initial magnetic perturbations, and when the geometry of the flow is such that an amplification mechanism can occur.

Recent experimental, analytical and numerical investigations [4, 7-10] suggest that large-scale fluctuations of the flow constitute an obstacle to dynamos, which are sometimes not observed experimentally at Reynolds numbers where they are theoretically expected [11]. Strategies are needed in order to overcome this problem, and turbulence control is considered to be the key action to take [10].

We study numerically dynamo action in a turbulent flow that is stirred by a large-scale, constant axisymmetric force in spherical geometry, as in the Madison Dynamo Experiment (MDE) [11]. The force creates a two-vortex flow around the $z$ axis (the so-called $s 2 t 2$ flow), previously studied numerically by Dudley and James [15] who suggested it as a flow which optimizes the magnetic field generation. We carried out simulations using the DYNAMO code [16, 17], a pseudo-spectral code which mimics the conditions of the MDE, in order to identify the vortices with possible negative effects, characterized their scale and their temporal evolution, and finally proposed practical strategies whereby these vortices may be controlled experimentally.

In order to understand which configurations are detrimental for the dynamo process, we analyze the simulated velocity fields via Singular Value Decomposition (SVD), a mathematical method used in order to simplify the representation of datasets (see [18] or [19] for its applications to fluid dynamics). SVD reduces the number of variables necessary to quantify the properties of the data under analysis: One can limit oneself to analyzing only the information described typically by the first few new variables. A generic spatio-temporal field
$A$ is sampled over $N_{\text {space }}$ spatial points and $N_{\text {time }}$ timeshots, i.e., $A_{i j}=A\left(\mathbf{r}_{i}, t_{j}\right)\left(i=1, \ldots, N_{\text {space }} ; j=1, \ldots, N_{\text {time }}\right)$. It is then decomposed via the SVD theorem,

$$
A_{i j}=\sum_{k=1}^{N_{S V D}} \sigma_{k} u_{k}\left(\mathbf{r}_{i}\right) v_{k}\left(t_{j}\right)
$$

where $N_{S V D}=\min \left(N_{\text {space }}, N_{\text {time }}\right)$.

The spatial fields $u_{k}\left(\mathbf{r}_{i}\right)$ are the new generalized basis functions and the temporal functions $\sigma_{k} v_{k}\left(t_{j}\right)$ can be seen as the temporal evolution of the amplitude of the $k$-th mode. The "singular values" $\sigma_{k}$ are ordered in descending order of magnitude: $\sigma_{1}>\sigma_{2}>\ldots>\sigma_{N_{S V D}}$; they quantify the weight of the associated $k$-th mode relative to the other modes, i.e., the amount of information (closely related to the energy) contained in it. In the following, the fields $u_{k}\left(\mathbf{r}_{i}\right)$ will be called the SVD modes.

Simulation setup.- The DYNAMO code solves the NavierStokes equation coupled with the magnetic induction equation, which, in its nondimensional form, reads

$$
\frac{\partial \mathbf{B}}{\partial t}=\operatorname{Rm} \nabla \times(\mathbf{v} \times \mathbf{B})+\nabla^{2} \mathbf{B},
$$

where $\mathrm{Rm}=\mu_{0} \sigma R U$ is the magnetic Reynolds, $R$ the characteristic length associated with the system (the radius of the sphere), $U$ the characteristic amplitude of the velocity field $\mathbf{v}$, and $\sigma$ the conductivity of the medium. We apply the SVD analysis on the spectral representation of the velocity fields produced by hydrodynamical simulations (i.e., setting to zero the conductivity of the medium) whose numerical fluid Reynolds numbers are $\operatorname{Re}_{0}=\{600,1100,3000\}$. $\operatorname{Re}_{0}$ is a parameter read by the DYNAMO code before a simulation is started; it is multiplied with the characteristic velocity $U$ to obtain the real fluid Reynolds number Re.

The number of radial grid points in the sphere is $n_{r}=512$. The DYNAMO code uses a spherical harmonics decomposition to handle the $\theta$ and $\phi$ dependencies of the fields: We study three simulations with spectral resolution of $l_{\max }=$ $\{52,52,180\}$, respectively, where $l_{\max }$ is the maximum "angular momentum" wavenumber. The velocity fields are decomposed into $n_{\text {modes }}=\left(l_{\max }+1\right)\left(l_{\max }+2\right) / 2-1$ modes [21]. We 
analyze, respectively, $N_{\text {time }}=\{1550,700,400\}$ snapshots of the velocity field after the transient phase. The numbers $N_{\text {time }}$ are chosen according to the available computer resources. We represent the spatio-temporal DYNAMO datasets (i.e., the spectral components of the velocity field) as an $N_{\text {space }} \times N_{\text {time }}$ matrix, where $N_{\text {space }}$ is $2 n_{r} n_{\text {modes }}$ (the prefactor 2 due to the presence of a toroidal and a poloidal component for every spherical harmonic mode, see [21]).

Singular values.- The decay of the singular values $\sigma_{k}$ is exponential in the medium range of $k$ modes and it is even steeper at low $k$, i.e., the information on the dynamics is condensed in the first SVD modes. Table I reports the percentage of information content of the first $6 k$ modes calculated via the definition of $p_{k}=\sigma_{k}^{2}\left(\sum_{k=1}^{N_{S V D}} \sigma_{k}^{2}\right)^{-1}$ (i.e., the relative amount of information contained in the $k$-th mode, see above). At higher $\mathrm{Re}_{0}$, the flow becomes progressively more turbulent and, comparatively, more energy is drained from the mean field and stored in the other extraneous modes.

TABLE I: Information content of the first $6 k$ modes calculated via $p_{k}$.

\begin{tabular}{ccccccc}
\hline \hline & $k=1$ & $k=2$ & $k=3$ & $k=4$ & $k=5$ & $k=6$ \\
\hline $\operatorname{Re}_{0}=600$ & $66.6 \%$ & $9.6 \%$ & $5.4 \%$ & $2.9 \%$ & $2.4 \%$ & $1.8 \%$ \\
$\operatorname{Re}_{0}=1100$ & $56.7 \%$ & $16.7 \%$ & $5.1 \%$ & $3.6 \%$ & $2.6 \%$ & $1.7 \%$ \\
$\operatorname{Re}_{0}=3000$ & $54.9 \%$ & $15.2 \%$ & $5.3 \%$ & $4.1 \%$ & $2.5 \%$ & $1.9 \%$ \\
\hline
\end{tabular}

The first two SVD modes.- The first SVD mode, $u_{1}(\mathbf{r})$, broadly captures the essential features of this hydrodynamic virtual experiment, being very similar to the mean field, which in turn resembles the $s 2 t 2$ input flow.

The second SVD eigenfunction, $u_{2}(\mathbf{r})$, is still relatively important (see Table I). It consists of three main components: (a) a toroidal vortex (which shares the symmetry of the impellers) with a strong activity at $\rho \sim 0.14 R$ ( $R$ is the radius of the sphere, $\rho$ is in cylindrical coordinates); (b) a poloidal circulation which provides the velocity field with a vertical component; this component has a relatively strong amplitude at about the same cylindrical distance from the $z$ axis as the toroidal vortex, making $u_{2}(\mathbf{r})$ helical; (c) noisy toroidal and poloidal components with negligible amplitudes. In other words, the vortex has a smooth and large-scale spatial dependence (see Figures 1 and 2), with an elongated helical shape oriented along the axis of symmetry of the forcing mechanism. It does not show any counter-rotating feature, as the $s 2 t 2$ flow; on the contrary, its "winding" configuration is equal in the two hemispheres.

Note that this flow is characterized, at first glance, by a puzzling breaking of the symmetry expected from the driving mechanism; the flow is driven by counter-rotating impellers, and yet $u_{2}(\mathbf{r})$ is characterized by a uniform rotation orientation on the axis between the impellers. The expected symmetry can only be observed - in an average sense - when one follows the temporal evolution of the mode, which exhibits alternating phases of opposite rotational orientation (see below).

The presence (and the fluctuations) of these secondary flow

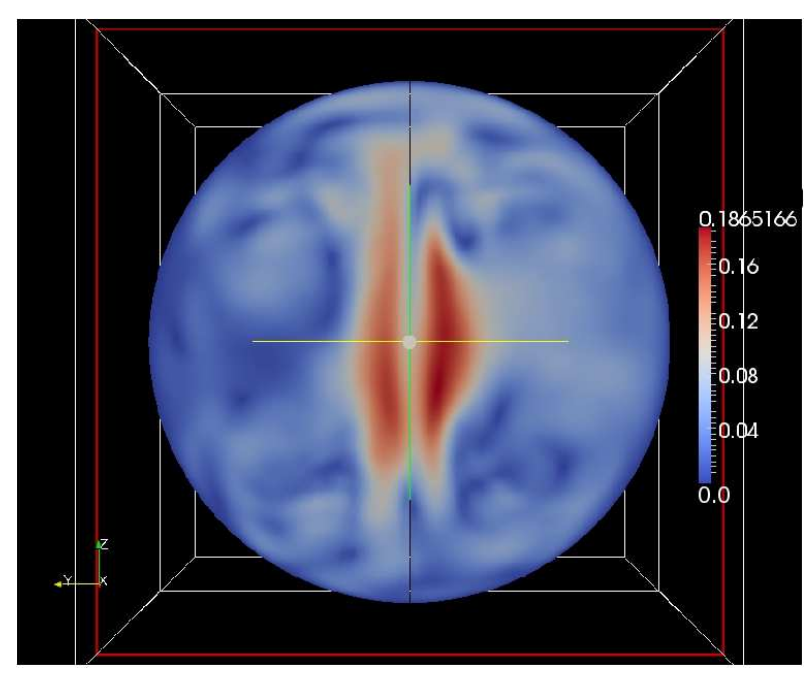

FIG. 1: (Color online) Cross section of the sphere, coordinate plane $y z$. The color represents the magnitude of the velocity field of the second SVD mode, $\operatorname{Re}_{0}=600$. The vortex has its strongest activity along the axis of symmetry, at a distance of $\sim 0.14$.

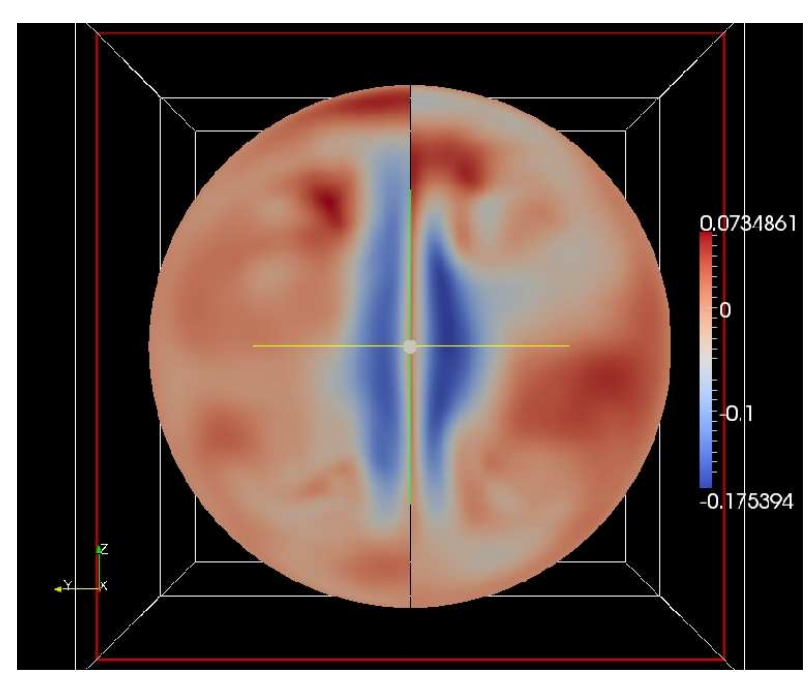

FIG. 2: (Color online) Cross section of the sphere, coordinate plane $y z$. The color represents the $\phi$ component of the velocity field of the second SVD mode, $\mathrm{Re}_{0}=600$. The color changes discontinuously from $y<0$ to $y>0$ due to the fact that the $y$-component of the unit vector $\hat{e}_{\phi}$ changes sign form $y>0$ to $y>0$.

can extract energy from the growing magnetic mode and inject it into damped magnetic modes, hampering the dynamo instability, e.g., via a non-linear three-mode interaction similar to that described in [10].

Temporal evolution of the first two SVD modes.- The SVD analysis provides a detailed description of the dynamics of the $u_{k}(\mathbf{r})$ modes: The temporal eigenfunctions $\sigma_{1} v_{1}(t)$ and $\sigma_{2} v_{2}(t)$ describe the (decoupled) dynamics of the associated spatial modes. As can be seen in Figures 3 and 4, the imaginary parts of the two time series are negligible compared to the real parts. The real part of $v_{1}(t)$ oscillates around a sta- 
ble value which never crosses the $\sigma_{1} v_{1}(t)=0$ axis: As we expect from the constancy of the force, the dynamics of the velocity field is basically a constant $s 2 t 2$ flow with a superimposed turbulent variation of the local magnitude of the vector field, conserving the rotational direction in each hemisphere. The behavior of $\sigma_{2} v_{2}(t)$ is completely different: The real part changes sign, i.e., the helical vortex alternates phases with opposite rotational directions. These dynamics are difficult to extract without the use of SVD, which constructs basis functions specifically suited to the problem, and also clearly elucidates the temporal dynamics of each basis function.

The dynamics of this hydrodynamic simulation can be described by the following scenario. The usual counter-rotating $s 2 t 2$ flow is produced by the forcing mechanism and it constitutes the main component of the flow, i.e., a stationary background flow. Superimposed upon this flow, a vortex-like component is spread over the axis of symmetry, with an alternating activity between two opposed configurations, one with the same rotation direction of the $s 2 t 2$ flow in the northern hemisphere, the other one in the southern hemisphere. The additivity property of the decomposition states that the field associated with this secondary motion reinforces the magnitude of the velocity in the northern (southern) hemispheres and weakens the other one, when it is in the former (latter) configuration: In addition to the expected $s 2 t 2$ background flow, each hemisphere has a pulsating activity in counter-phase respect to the other.

It is interesting to note that a similar behavior has been obtained experimentally for a turbulent von Kármán swirling flow, where two impellers counter-rotate inside a cylindrical cavity [12-14]. The flow shows two configurations, each one characterized by a "dominant cell" (alternatively, the north or the south cell) with a higher velocity and a larger spatial extent, comparable to the "dominant hemisphere" picture described above (although the experimental Re can reach values of $10^{6}$ ).

Role of time-stationary $u_{2}(\mathbf{r})$ in the dynamo process.- In order to identify the impact of $u_{2}(\mathbf{r})$ on the dynamo, we performed three kinds of time-stationary kinematic (i.e., the flow does not evolve and is decoupled from the magnetic field) simulations at $\operatorname{Re}_{0}=600$ and different $\mathrm{Rm}$. The first one uses as fluid flow only $u_{1}(\mathbf{r})$ (the dominant mode), weighted with the mean value $\left\langle\sigma_{1} v_{1}(t)\right\rangle$ (see Fig. 3). The second (third) run uses the superposition of $u_{1}(\mathbf{r})$ with $u_{2}(\mathbf{r})$, having weighted $u_{2}(\mathbf{r})$ with the maximum positive (minimum negative) value of the oscillating time trace. The impact on the dynamo process can be summarized by comparing the growth rates of the magnetic energy (Table II and Fig. 5, runs indicated symbolically as "1", "1+2", "1-2", respectively).

As Fig. 5 shows, a time-stationary $u_{2}(\mathbf{r})$ has a detrimental effect when coupled to the first SVD mode at $\operatorname{Re}_{0}=600$. At $\mathrm{Re}_{0}=1100, u_{1}(\mathbf{r})$ is noisier and less axisymmetric since the temporal sampling is shorter (the resolution is higher and the dataset should be reduced in order to apply the SVD with the same CPU resources): adding and subtracting $u_{1}(\mathbf{r})$ and $u_{2}(\mathbf{r})$ produces different values of $\gamma$ because the noisy toroidal com-

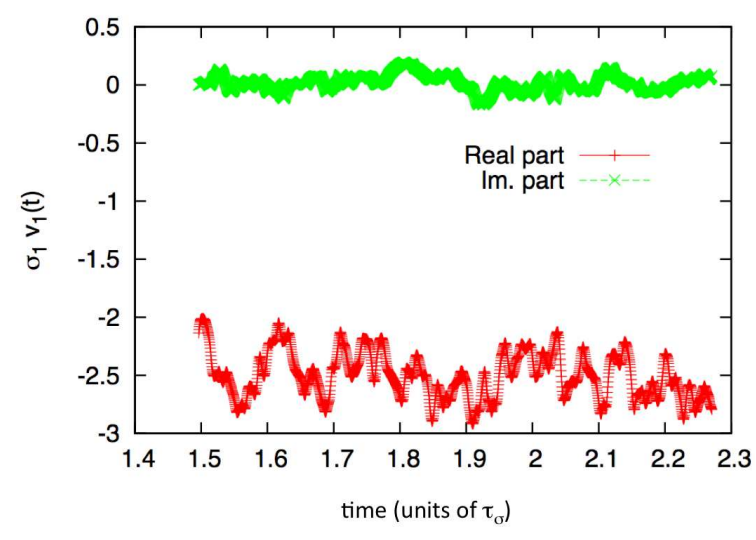

FIG. 3: (Color online) Real and imaginary parts of the SVD temporal eigenfunction $v_{1}(t)$.

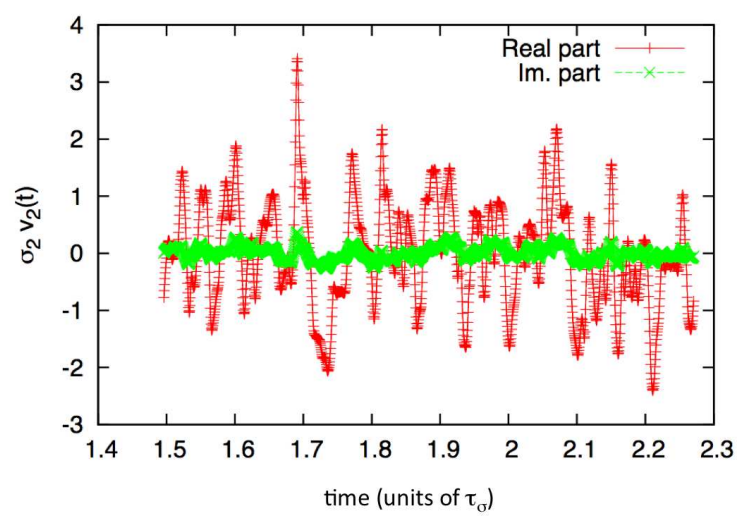

FIG. 4: (Color online) Real and imaginary parts of the SVD temporal eigenfunction $v_{2}(t)$.

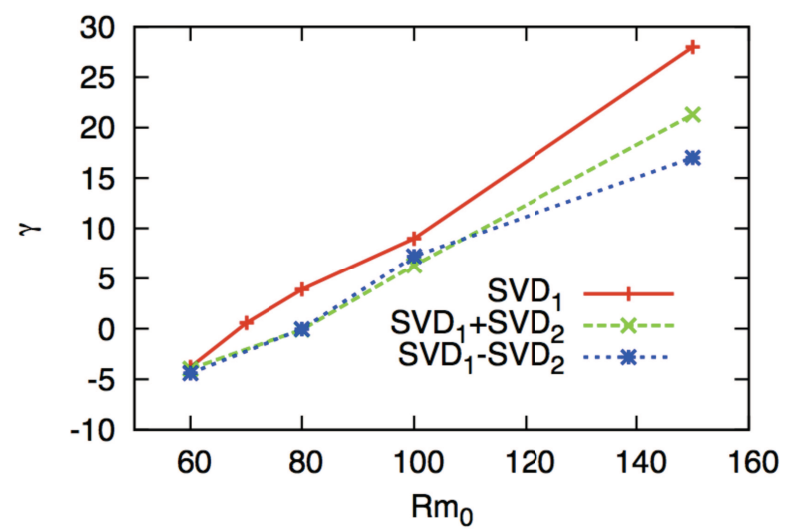

FIG. 5: (Color online) Growth rates of $E_{M}$ for the runs "1", "1+2", and " $1-2 ", \operatorname{Re}_{0}=600$.

ponent of $u_{1}(\mathbf{r})$ tends to cancel out $u_{2}(\mathbf{r})$ in the "1-2" configuration. On the other hand, in the " $1+2$ " configuration, $u_{2}(\mathbf{r})$ turns out to be detrimental. This last analysis does not take into consideration the role of the fluctuations in $u_{2}(\mathbf{r})$, which play a detrimental role, as suggested by the three-wave turbulent interaction picture in [10]. 
TABLE II: Growth rates of $E_{M}$ for the runs "1" and " $1+2$ ", $\mathrm{Re}_{0}=$ 1100 .

\begin{tabular}{lcc}
\hline \hline & & \\
& "1" $1+2 "$ \\
\hline $\mathrm{Rm}_{0}=100$ & $5.64 \pm 0.013$ & $-3.8 \pm 0.4$ \\
$\mathrm{Rm}_{0}=150$ & $27.4 \pm 0.4$ & $5.1 \pm 0.5$ \\
\hline
\end{tabular}

An interpretation of the $\mathrm{Rm}_{c}(\mathrm{Re})$ curve.- One of the main results of [22] is the study of the stability curve $\operatorname{Rm}_{c}(\mathrm{Re})$ of this system, where $\mathrm{Rm}_{c}$ is the critical magnetic Reynolds number (above which the dynamo instability takes place). The underlying physical mechanism of this curve can be explained in more detail in the light of the results of this work. Table I shows that the relative energy content of the vortices with a negative effect is enhanced by turbulence, whereas the energy in the mean flow would still depend only on the largescale force (and not on Re). In fact, the presence of $u_{2}(\mathbf{r})$ at $\mathrm{Re}_{0}=600$ enhances $\mathrm{Rm}_{c}$ by $20 \%$, whereas at $\mathrm{Re}_{0}=1100$ by $37 \%$. The picture suggests that turbulence, increasing the energy of $u_{n}(\mathbf{r})$ (with $n \geq 2$ ) and not of $u_{1}(\mathbf{r})$ - which is prescribed by the force - makes the dynamo instability more difficult to reach, as the quasi-linear behavior of the $\mathrm{Rm}_{c}(\mathrm{Re})$ curve shows at low Re. On the other hand, the saturation of the curve at high Re can be explained by the fact that the modes at smaller scales are not allowed to grow indefinitely at high $\mathrm{Re}_{0}$ without a specific small-scale force.

A possible strategy for enhancing the dynamo.- In order to suppress or manipulate these detrimental dynamics, we implemented in the code the effect of a discoidal baffle on the equatorial plane (a circular plate in the middle of the plane). Kinematic simulations show that the growth rate $\gamma$ of $E_{M}$ is strongly enhanced by such a disk, whose radius $r_{d}$ was chosen in order to cover spatially at least the region of the equatorial plane through which $u_{2}(\mathbf{r})$ flows. Table III shows the effect of the disk on $\gamma$. A larger $r_{d}$ is needed to have a non negligible effect at $\operatorname{Re}_{0}=1000$, confirming the necessity of a stricter control of large-scale turbulence at higher $\operatorname{Re}_{0}$. The value $\gamma_{r}$ represents the growth rate of $E_{M}$ in the presence of a outer ring on the equatorial plane, as implemented recently in the MDE and used in the past in the VKS experiment. The simulations show that the ring effect does not improve the dynamo efficiency as the disc does.

Kinematic simulations show that the growth rate $\gamma$ of $E_{M}$ is enhanced by both the ring and the disc. Nevertheless, the ring effect is not as strong as the disc effect. Table III shows the effect of the disc on the default growth rate $\gamma$. We indicate with $\gamma_{r}$ the growth rate of $E_{M}$ in presence of the ring, $\gamma_{d}$ in presence of the disc. It should be noted that a larger $r_{d}$ is needed to have a non negligible effect at $\operatorname{Re}_{0}=1000$, confirming the necessity of a stricter control of large scale turbulence at higher $\operatorname{Re}_{0}$.

This last analysis suggests how to put into practice these results: controlling the symmetry around the equator of the counter-rotating flow, avoiding a strong poloidal circulation near the axis and facilitating the separation of the dynamics of the toroidal circulation in the two hemispheres can be the
TABLE III: Kinematic simulations of dynamos with an implemented equatorial disc in the center of the equatorial plane. $\gamma_{d}$ is the growth rate (the time is scaled to the resistive diffusion time, $\tau_{\sigma}=\mu_{0} \sigma L^{2}$ ) of $E_{M}$ in the presence of the disk; $\gamma_{r}$ in the presence of the ring; $\gamma_{0}$ is the default growth rate, i.e., without any baffle.

\begin{tabular}{ccccccc}
\hline \hline $\mathrm{Re}_{0}$ & $\mathrm{Rm}_{0}$ & $l_{\max }$ & $r_{d}$ & $\gamma_{r}$ & $\gamma_{d}$ & $\gamma_{0}$ \\
\hline 300 & 80 & 30 & 0.28 & 7.62 & 18.8 & 4 \\
300 & 100 & 30 & 0.28 & 15.5 & 30.8 & 9.8 \\
300 & 150 & 30 & 0.28 & 31.2 & 58.6 & 24 \\
300 & 250 & 30 & 0.28 & 52 & 109 & 39 \\
600 & 250 & 52 & 0.28 & 28.4 & 45.4 & 22.6 \\
600 & 250 & 72 & 0.28 & 29.9 & 50.4 & 22.6 \\
1000 & 300 & 52 & 0.65 & 10.4 & 18.4 & 10.4 \\
\hline
\end{tabular}

right strategy for facilitating the dynamo instability.

Summary.- In this Letter, we have reported about hydrodynamic simulations in the context of dynamo theory in a flow geometry important for laboratory dynamos. We have shown that a mean counter-rotating flow develops a smalleramplitude, large-scale turbulent helical vortex oriented along the symmetry axis of the forcing mechanism. This vortex has a spatio-temporal behavior which has been observed experimentally in a similar topology: It does not counter-rotate and its rotational orientation changes in time, enforcing the stirring mechanism in only one hemisphere at a time. These secondary dynamics turn out to be detrimental for dynamo purposes. Further MHD simulations suggest experimental methods for facilitating dynamo excitation through manipulation of this helical flow.

The computations were performed on the BOB and TOK clusters hosted at the Garching Computing Center (RZG) of the Max Planck Society.

* Electronic address: angl@ipp.mpg.de

[1] G. Rüdiger and R. Hollerbach, The Magnetic Universe, WileyVCH (2004).

[2] H. K. Moffatt, Magnetic Field Generation in Electrical Conducting Fluids, Cambridge University Press (1978).

[3] R. Stieglitz and U. Müller, Phys. Fluids 13, 561 (2001).

[4] F. Pétrélis and S. Fauve, Europhys. Lett. 76, 602 (2006)

[5] E. Dormy and A. M. Soward, Mathematical Aspects of Natural Dynamos, Chapman \& Hall/CRC (2007).

[6] A. Gailitis et al. Comptes Rendus Physique 9, 7 (2008).

[7] J. P. Laval, P. Blaineau, N. Leprovost, B. Dubrulle, F. Daviaud, Phys. Rev. Lett. 96, 204503 (2006).

[8] Y. Ponty et al., New J. Phys. 9, 296 (2007).

[9] K. Reuter et al., New J. Phys. 11, 013027 (2009).

[10] E. J. Kaplan et al., Phys. Rev. Lett. 106, 254502 (2011).

[11] C. B. Forest et al., Magnetohydrodynamics 38, 107 (2002).

[12] A. de la Torre and J. Burguete, Phys. Rev. Lett. 99, 054101 (2007).

[13] E. Crespo Del Arco et al., Geophys. \& Astrophys. Fluid Dyn. 103, 163-177 (2009).

[14] A. de la Torre et al., The European Physical Journal - Special Topics, 146, 313-320 (2007). 
[15] M. L. Dudley and R.W. James, Proc. R. Soc. Lond. 425, 407 (1989).

[16] R. A. Bayliss, C.B. Forest, M.D. Nornberg, E.J. Spence, P.W. Terry, Phys. Rev. E 75, 026303 (2007).

[17] K. Reuter et al., Computer Physics Communications 179, 245 (2008).

[18] S. Futatani et al., Phys. Plasmas 16, 042506 (2009).

[19] G. Berkooz et al., Annu. Rev. Fluid Mech 25, 539 (1993).
[20] E. Bullard, H. Gellman, Philosophical Transactions of the Royal Society of London 247, 213 (1954).

[21] K. Reuter, Numerical investigations of turbulent dynamo excitation in a spherical MHD system, Westfälische WilhelmsUniversität Münster (2010).

[22] K. Reuter et al., New J. Phys. 13, 073019 (2011). 